\title{
Sensitivity of $p$-mode absorption on magnetic region properties and kernel functions
}

\author{
A. Gascoyne ${ }^{1}$, R. Jain ${ }^{1}$, and B. W. Hindman ${ }^{2}$ \\ 1 Applied Mathematics Department, The University of Sheffield, S3 7RH, UK \\ e-mail: [app07adg;R.Jain]@sheffield.ac.uk \\ 2 JILA, University of Colorado at Boulder, USA \\ e-mail: hindman@lcd.colorado.edu
}

Received 8 October 2010 / Accepted 1 December 2010

\begin{abstract}
Aims. Magnetohydrodynamic (MHD) sausage tube waves are excited in magnetic flux tubes by p-mode forcing. These tube waves carry energy away from the p-mode cavity which results in a source of absorption. We wish to see the effect of an ensemble of randomly distributed thin magnetic flux tubes on the absorption of p-modes for the model plage region and also study the effect of the spacial weighting function on the theoretically calculated absorption coefficients.

Methods. We calculate the absorption coefficients of $\mathrm{p}$ modes for a model plage, assumed to consist of an ensemble of many thin magnetic flux tubes with randomly distributed plasma properties. Each magnetic flux tube in the ensemble is modelled as axisymmetric, non-interacting, vertically oriented and untwisted.

Results. We find that the magnitude and the form of the absorption coefficient is sensitive to the plasma- $\beta$ of the tubes which is consistent with previous work. Both the random distribution used to model the ensemble of flux tubes and the spatial weighting function inherent to the measurement of the absorption affect the absorption. As the width of the weighting function increases, the absorption increases.
\end{abstract}

Key words. Sun: helioseismology - Sun: oscillations - magnetic fields - magnetohydrodynamics (MHD) - scattering

\section{Introduction}

Solar acoustic modes ( $\mathrm{p}$ modes) are absorbed and scattered by magnetic inhomogeneities such as sunspots or plage regions. Scattering by such structures has been studied, using various observational helioseismic techniques (see Braun et al. 1988; Couvidat et al. 2006). Braun \& Birch (2008) found that sunspots can absorb over half of incident $\mathrm{p}$-mode power and plage regions can absorb around 20\%. The measured "bell-shaped" absorption has a well-established frequency dependence; the absorption rises from zero at zero frequency, plateaus around $4 \mathrm{mHz}$ and then suffers a precipitous drop at higher frequencies; this drop off has now been deemed unphysical due to local emission at higher frequencies (Braun \& Birch 2008).

This "bell-shaped" absorption had been known for decades (e.g., Braun et al. 1988) and theoretical models have been constructed to interpret the frequency variation in an attempt to understand the sub-structure of these magnetic regions. One proposed mechanism is mode conversion. Classical MHD wave theory reveals that where fast and slow magnetoacoustic waves share the same phase velocity (i.e., at the equipartition layer), transformation of acoustic wave energy occurs between these two waves (see, for example Crouch \& Cally 2003). Other mechanisms include resonant absorption (Hollweg 1988), mode mixing (D'Silva 1994) and excitation of tube waves through p-mode buffeting (Bogdan et al. 1996; Hindman \& Jain 2008).

Spruit (1981) derived the governing equations for two basic modes of oscillation for a thin magnetic flux tube: kink modes, which describe transverse, incompressive motions of the tube supported by magnetic tension, and sausage modes, which are longitudinal, compressive motions driven by the external pressure perturbations at the boundary between the tube and the nonmagnetic medium. The excitation of tube waves through p-mode buffeting was first introduced in Spruit (1991) and further examined in Bogdan et al. (1996). Bogdan et al. (1996) calculated pmode damping rates for such a mechanism using a photospheric stress-free boundary condition thus only allowing energy to be lost down the tube as the waves are reflected at the surface. To combat this issue of what surface boundary condition to use, Hindman \& Jain (2008) introduced a maximal-flux condition whereby no reflection takes place at the surface, allowing energy to propagate above the surface. This boundary condition therefore, gives the other extreme of the stress-free condition, i.e., it places an upper limit on the energy lost to the solar atmosphere. Jain et al. (2009) studied this mechanism to calculate the absorption of $\mathrm{p}$ modes in a simulated plage region, comparing with observational results from Braun \& Birch (2008).

In this paper we wish to study two different effects: how do details of the observational kernel function which represents the spatially weighted average of the magnetic flux around a point, modify the measured absorption coefficient and how does a distribution of tube parameters (i.e., plasma- $\beta$ ) influence the physical absorption. We construct a model consistent with Jain et al. (2009) in order to simulate the absorption of a plage region, with a random collection of thin, axisymmetric, vertical magnetic flux tubes. In Sect. 2 we describe the equilibrium configuration of our magnetic flux tubes, which are embedded in an adiabatically stratified, plane-parallel, polytropic atmosphere. In 
Sect. 3 we construct a wave field for our model. In Sect. 4 we describe the absorption mechanism. We present our results and discuss our findings in Sect. 5 and conclude in Sect. 6.

\section{Equilibrium configuration}

We consider magnetic fibril fields as axisymmetric magnetic flux tubes embedded in a plane-parallel, gravitationally stratified atmosphere. We wish to study the excitation of magnetohydrodynamic (MHD) tube waves through the buffeting of the tubes by external acoustic oscillations residing beneath the solar photosphere.

\subsection{Background atmosphere}

We model the nonmagnetic medium as a polytropic atmosphere with constant gravity acting downwards $\boldsymbol{g}=-g \hat{z} ; z$ is increasing upwards. The pressure, density and sound speed vary as power laws as follows:

$P_{\text {ext }}(z)=\frac{g z_{0} \rho_{0}}{a+1}\left(-\frac{z}{z_{0}}\right)^{a+1}=P_{0}\left(-\frac{z}{z_{0}}\right)^{a+1}$,

$\rho_{\mathrm{ext}}(z)=\rho_{0}\left(-\frac{z}{z_{0}}\right)^{a}$,

$c_{\mathrm{ext}}^{2}(z)=-\frac{g z}{a}$,

where the subscript " 0 " refers to the value at the model photosphere. Following Bogdan et al. (1996), Hindman \& Jain (2008) and Jain et al. (2009) we set the truncation depth at $z=-z_{0}$ which is the model photosphere where the quantities $\rho_{0}$ and $P_{0}$ are the values of the mass density and gas pressure at this depth. Above the truncation depth $z>-z_{0}$ we assume the existence of a hot vacuum $\left(\rho_{\text {ext }} \rightarrow 0\right.$ with temperature $\left.T_{\text {ext }} \rightarrow \infty\right)$. The external pressure, density and sound speed increase with depth and as our atmosphere is in convective equilibrium, the polytropic in$\operatorname{dex} a$ is related to the ratio of specific heats $\gamma$ via $a=1 /(\gamma-1)$. We also set the following characteristic physical scales for our model photosphere, $\rho_{0}=2.78 \times 10^{-7} \mathrm{~g} \mathrm{~cm}^{-3}, P_{0}=1.21 \times$ $10^{5} \mathrm{~g} \mathrm{~cm}^{-1} \mathrm{~s}^{-1}$, and $g=2.775 \times 10^{4} \mathrm{~cm} \mathrm{~s}^{-1}$, which coincide with the photospheric reference model of Maltby et al. (1986). The choice of polytropic index $a=1.5$ yields the truncation depth $z_{0}=392 \mathrm{~km}$ and a photospheric sound speed of $8.52 \mathrm{~km} \mathrm{~s}^{-1}$.

\subsection{Slender magnetic tube configuration}

We assume the magnetic field is comprised of thin magnetic flux tubes with the following properties:

- vertically aligned with the $z$-axis;

- untwisted and axisymmetric;

- embedded inside the field-free polytropic atmosphere;

- potential magnetic field, thus force free (i.e., no internal currents);

- thin such that the tube's radius is much smaller than any characteristic length scale such as the atmospheric scale lengths and the wavelength of tube waves.;

- flux tube interior has the same temperature variation with depth as the surrounding polytrope.

For such a thin magnetic flux tube to be in hydrostatic and thermodynamic balance requires that temperature and total pressure are uniform across the tube and equal to the external values. Thus the magnetic pressure has the same scale height as the gas pressure, which results in a constant plasma- $\beta$ with height within the tube. Since the gas pressure decreases rapidly with height in the solar atmosphere so does the magnetic pressure; this then results in significant flaring of field lines which inevitably breaks the thin flux tube approximation. This is the reason for truncating the polytrope at a depth which ensures that the thin flux tube approximation is valid. The radial variation of the magnetic field is ignored (see Bogdan et al. 1996, for details). Thus the tube's internal pressure $P(z)$, density $\rho(z)$, magnetic field $B(z)$, and crosssectional area $A(z)$ can be described as follows,

$$
\begin{aligned}
& P(z)=\frac{\beta}{\beta+1} P_{\text {ext }}(z), \\
& \rho(z)=\frac{\beta}{\beta+1} \rho_{\text {ext }}(z), \\
& \frac{B^{2}(z)}{8 \pi}=\frac{1}{\beta+1} P_{\text {ext }}(z), \\
& A(z)=\frac{\theta}{B(z)}=\left(\frac{\beta+1}{8 \pi P_{\text {ext }}(z)}\right)^{1 / 2} \theta .
\end{aligned}
$$

It is important here to point out the relationship between $A(z)$, $B(z)$ and $\theta$ for a single tube. For any given flux tube we are free to specify only two of the four parameters: the photospheric radius $R_{0}$ (or cross-sectional area $A_{0}=\pi R_{0}^{2}$ ), the magnetic flux $\theta$, the photospheric field strength $B_{0}$ and the plasma- $\beta$. Once specified, these two are the two remaining parameters are determined by Eqs. (6) and (7).

The next step is to simulate a plage region by considering a random distribution of flux tubes. We choose to restrict our attention to a range of $\beta$ values between 0 and 2, spanning the strong to weak field regimes. We shall use three different random distributions, a uniform distribution so all $\beta$ values have equal probability, thus mean $\beta=1$. We shall also consider a normal (Gaussian) distribution of flux tubes with mean $\beta=1$ and variance 1 (or e-folding length of 1 ). The final distribution we shall take is an exponential distribution such that the probability decreases as $\beta$ increases with mean $\beta=0.2$ and variance 1 . For simplicity we shall assume that every individual tube has equal magnetic flux $\theta$. This means that changing $\beta$ has the effect of changing the radius of the tube (see Eq. (7)).

\section{Wavefield description}

The field-free atmosphere supports resonant acoustic oscillations (f and p modes) that are trapped in a waveguide below the model photosphere. The upper boundary of the waveguide is the photosphere itself while the lower boundary is refractive, arising from the linear increase in temperature with depth.

\subsection{Wave equations}

In the field-free atmosphere, for wave perturbations $p_{\mathrm{ext}}^{\prime}, \rho_{\mathrm{ext}}^{\prime}$, $\xi^{\prime}$, in pressure, density and fluid displacement, respectively, we have the following linearized hydrodynamic equations,

$\rho_{\mathrm{ext}}^{\prime}+\xi_{\|}^{\prime} \frac{\partial \rho_{\mathrm{ext}}}{\partial z}+\rho_{\mathrm{ext}}\left(\boldsymbol{\nabla} \cdot \boldsymbol{\xi}^{\prime}\right)=0$,

$p_{\mathrm{ext}}^{\prime}+\xi_{\|}^{\prime} \frac{\partial P_{\mathrm{ext}}}{\partial z}+\gamma P_{\mathrm{ext}}\left(\boldsymbol{\nabla} \cdot \boldsymbol{\xi}^{\prime}\right)=0$ 
$\rho_{\mathrm{ext}} \frac{\partial^{2} \boldsymbol{\xi}^{\prime}}{\partial t^{2}}+\nabla p_{\mathrm{ext}}^{\prime}-\boldsymbol{g} \rho_{\mathrm{ext}}^{\prime}=0$

where $\xi_{\|}^{\prime}=\xi^{\prime} \cdot \hat{z}$. By eliminating $p_{\mathrm{ext}}^{\prime}$ and $\rho_{\mathrm{ext}}^{\prime}$ and substituting $\xi^{\prime}=\nabla \Phi$ where $\Phi$ is the displacement potential we obtain the following partial differential equation,

$\frac{\partial^{2} \Phi}{\partial t^{2}}=c^{2} \nabla^{2} \Phi-g \frac{\partial \Phi}{\partial z}$

Here we have dropped the subscript on $c_{\text {ext }}^{2}$ because, as mentioned earlier, the temperature variation inside and outside the tube is the same.

The incident acoustic wavefield expressed by Eq. (11) supports plane wave solutions of the form,

$\Phi(\boldsymbol{x}, t)=\mathcal{A} e^{i(k x-\omega t)} Q(z)$,

where $\mathcal{A}$ is the complex wave amplitude, $\omega$ the temporal frequency, and $k$ the horizontal wavenumber. $Q(z)$ is the vertical eigenfunction. Substituting Eq. (12) into (11) generates the following ordinary differential equation

$\left[c^{2} \frac{\mathrm{d}^{2}}{\mathrm{~d} z^{2}}-g \frac{\mathrm{d}}{\mathrm{d} z}+\left(\omega^{2}-k^{2} c^{2}\right)\right] Q(z)=0$.

\subsection{Boundary conditions}

Using the physical boundary conditions that the solution vanishes deep in the atmosphere and the Lagrangian pressure perturbations vanishes at the surface (i.e., at the truncation depth $z=-z_{0}$ ), the solutions to Eq. (13) form a set of orthogonal eigenfunctions,

$Q(z)=w^{-(\mu+1 / 2)} W_{\kappa, \mu}(w)$,

which depends on the following dimensionless parameters,

$w=-2 k z, \quad \mu=\frac{(a-1)}{2}, \quad v^{2}=\frac{a \omega^{2} z_{0}}{g}, \quad \kappa=\frac{v^{2}}{2 k z_{0}}$.

Vanishing Lagrangian pressure perturbation at the upper boundary $\left(\boldsymbol{\nabla} \cdot \boldsymbol{\xi}^{\prime}=0\right)$, at $z=-z_{0}$, requires the following quantization condition (see Bogdan et al. 1996 or Hindman \& Jain 2008, for details),

$W_{\kappa, \mu+1}\left(\frac{v^{2}}{\kappa}\right)=0$.

The eigenvalues $\kappa$ have a discrete set of values. For the special case of a "complete" polytrope, where $z_{0}=0$, the eigenvalues take the special form $\kappa_{n}=1 / 2+\mu+n$. In our more general case, with $z_{0} \neq 0$, the $\kappa_{n}$ must be generated numerically from the roots of Eq. (15).

\section{Absorption via the excitation of tube waves}

Here we consider the absorption of acoustic waves arising from the excitation of magnetic tube waves. We ignore the excitation of the acoustic jacket and the existence of mode mixing (see Bogdan \& Cally 1995, for a full investigation into the presence of laterally evanescent jacket modes and Gordovskyy et al. 2009, for an explanation of the role of mode mixing in p-mode absorption).

\subsection{Magnetic flux tube oscillations}

We are seeking waves on thin tubes that lack internal structure. Only three types of MHD waves satisfy this criterion: torsional Alfvén waves, longitudinal (sausage) waves and transverse (kink) waves. We ignore torsional Alfvén waves because the p-mode oscillations are irrotational when the atmosphere is adiabatically stratified and therefore do not couple with torsional waves. In this paper we will also ignore the kink waves and specifically concentrate on axisymmetric oscillations. Using the formulation of Jain et al. (2009), the fluid displacement due to the excitation of sausage waves within the tube can be described by the following equation,

$$
\begin{gathered}
\left(\frac{\partial^{2}}{\partial t^{2}}+\frac{2 g z}{2 a+\beta(1+a)} \frac{\partial^{2}}{\partial z^{2}}+\frac{g(1+a)}{2 a+\beta(1+a)} \frac{\partial}{\partial z}\right) \xi_{\|}= \\
\frac{(1+a)(\beta+1)}{2 a+\beta(1+a)} \frac{\partial^{3} \Phi}{\partial z \partial t^{2}} .
\end{gathered}
$$

These sausage waves $\xi_{\|}(z, t)$ can be described as axisymmetric pressure pulses that produce displacements that are primarily parallel to the magnetic field. The p-mode driving term appears on the right hand side of Eq. (16) through the displacement potential $\Phi$. This equation can be put in nondimensional form as,

$\left(\frac{\mathrm{d}^{2}}{\mathrm{~d} s^{2}}+\frac{\mu+1}{s} \frac{\mathrm{d}}{\mathrm{d} s}+\frac{v^{2} \epsilon}{s}\right) \xi_{\|}=\frac{\mathcal{A}}{z_{0}} f(s)$,

where,

$s=-\frac{z}{z_{0}}, \epsilon=\frac{2 a+\beta(1+a)}{2 a}, f(s)=-\frac{(1+a)(\beta+1)}{2 a} \frac{v^{2}}{s} \frac{\mathrm{d} Q(s)}{\mathrm{d} s}$.

The homogeneous version of Eq. (17) has the following solution,

$\psi_{\|}(s)=s^{-\mu / 2} H_{\mu}^{(1)}(2 v \sqrt{\epsilon s})$,

where $H$ is the Hankel function (Abramowitz \& Stegun 1964) and $\psi_{\|}(s)$ represents a downward propagating sausage wave and its complex conjugate $\psi_{\|}^{*}(s)$ represents the upward propagating wave.

\subsection{Absorption coefficient for a single tube}

In order to calculate the absorption of a single thin magnetic flux tube, we follow the calculation presented in Jain et al. (2009) and Hindman \& Jain (2008). Quoting their result for the absorption coefficient $\alpha$ due to the generation of sausage waves, we have

$$
\begin{gathered}
\alpha=\frac{\pi \beta}{2(2+\gamma \beta)(\beta+1)} \frac{\lambda^{a+1}}{v^{2} \mathcal{H}} \frac{A_{0}}{z_{0}^{2}}\left(\left|\Omega+\mathcal{I}^{*}\right|^{2}+|\mathcal{I}|^{2}-|\Omega|^{2}+\mathcal{S}\right), \\
\mathcal{S}=-\frac{(1+a)(\beta+1)}{2 a} v^{2} Q_{0}\left\{H_{\mu}^{(1)}(2 v \sqrt{\epsilon})\left(\Omega+\mathcal{I}^{*}\right)\right. \\
\left.+H_{\mu}^{(2)}(2 v \sqrt{\epsilon})\left(\Omega^{*}+\mathcal{I}\right)\right\}
\end{gathered}
$$

where $\lambda=v^{2} / \kappa$ and $\mathcal{H}$ is an integral associated with the lateral energy flux carried by any individual wave component (see Jain et al. 2009, Eq. (11)). The integral

$\mathcal{I}=\int_{1}^{\infty} s^{\mu+1} \psi_{\|}(s) f(s) \mathrm{d} s$, 
describes the interaction between the p mode and the sausage wave.

The parameter $\Omega$ in Eq. (19) represents the boundary condition (BC) at the surface $s=1$ ( or $z=-z_{0}$ ). Similar to Jain et al. (2009) we take two separate BC at the surface, a stress-free and a maximal-flux condition. As mentioned in Sect. 1, the stress-free BC has the physical interpretation that all upward propagating waves are reflected at the surface. Maximalflux BC can be interpreted as no wave reflection at the surface, thus all upward propagating waves escape into the upper atmosphere. For a detailed explanation of how to calculate $\Omega$ for these two BC see Hindman \& Jain (2008); here we simply quote their result,

$\Omega_{\mathrm{SF}}=i \frac{(1+a)(\beta+1)}{a \pi} v^{2} \frac{Q_{0}}{\mathcal{R}}-\frac{\mathcal{R}^{*}}{\mathcal{R}} \mathcal{I}$,

for stress-free BC where,

$\mathcal{R}=v \sqrt{\epsilon} H_{\mu+1}^{(1)}(2 v \sqrt{\epsilon})+(\beta+1)(\mu+1) H_{\mu}^{(1)}(2 v \sqrt{\epsilon})$,

and,

$\Omega_{\mathrm{MF}}=-\frac{(1+a)(\beta+1)}{2 a} v^{2} Q_{0} H_{\mu}^{(2)}(2 v \sqrt{\epsilon})$,

for maximal-flux BC.

\subsection{Absorption for a random ensemble of tubes}

We wish to apply our theory to model the absorption coefficient measured for a typical solar plage region by examining the effect of a large number of thin magnetic flux tubes. We thus assume that the tubes are sufficiently separated so as to neglect the effect of multiple scattering.

In order to compute the number of tubes $N$ required to estimate the collective absorption coefficient of a plage we follow Jain et al. (2009). We use a spatial weighting function or kernel $K$, which linearly relates the distribution of magnetic flux in the plage region, to the observed absorption. Let $\Theta(\boldsymbol{r}, \omega)$ be the amount of magnetic flux sampled by an observation,

$\Theta(\boldsymbol{r}, \omega)=\int K\left(\boldsymbol{r}^{\prime}-\boldsymbol{r}, \omega\right)\left|B\left(\boldsymbol{r}^{\prime}\right)\right| \mathrm{d} \boldsymbol{r}^{\prime}$,

where $K(\boldsymbol{r}, \omega)$ is the kernel, and as Jain et al. (2009) assumed,

$K(\boldsymbol{r}, \omega)=\mathrm{e}^{-k^{2}|\boldsymbol{r}|^{2} / \pi^{2}}$,

where $k\left(=\omega^{2} \frac{a}{2 \kappa g}\right)$ is the horizontal wavenumber. $N$, the number of flux tubes, is equal to the magnetic flux $\Phi$ sampled by the kernel function divided by the magnetic flux of an individual flux tube $\theta$,

$N=\frac{\Theta(\boldsymbol{r}, \omega)}{\theta}$.

It is thus clear that $N$ is dependent on the form of the spatial weighting function. We shall investigate the effect of various kernel functions by including a constant $C$ in the spatial weighting function $K$ as follows,

$K(\boldsymbol{r}, \omega)=\mathrm{e}^{-C k^{2}|\boldsymbol{r}|^{2} / \pi^{2}}$.

Increasing the value of $C$ narrows the width of the kernel function $K(\boldsymbol{r}, \omega)$ and decreases the magnetic flux $\Theta(\boldsymbol{r}, \omega)$ (see Eq. (24)), thereby reducing the number of tubes required (vice versa for decreasing $C$ ).
To calculate the total absorption $\alpha_{\text {tot }}$ for the various distributions of tubes as discussed earlier in Sect. 2.2 we generate a random collection of $N$ tubes, each with a different value of $\beta_{i}$ drawn from the desired probability distribution. The collective absorption is then,

$\alpha_{\mathrm{tot}}=\sum_{i=1}^{N} \alpha\left(\beta_{i}\right)$,

where $\alpha\left(\beta_{i}\right)$ is the absorption for a single tube and the sum is over flux tubes. For high values of $N$, tubes with nearly identical properties are treated in bulk to reduce the number of random numbers that need to be generated.

\section{Results and discussion}

In this section we present the results for theoretically calculated absorption coefficients of $p$ modes by the generation of magnetic tube waves in the convection zone through external p-mode buffeting. In particular, we investigate the effect of a distribution of flux tube plasma- $\beta$. We consider $\theta$ to be the same for every tube in this model.

\subsection{Effect of kernel function on the absorption coefficient}

It was previously noted in Jain et al. (2009) that a poor approximation for the observation kernel will give a poor approximation for the number of flux tubes necessary to simulate the plage region. Here we wish to explicitly examine the effect of various constants $C$ in the Gaussian kernel function on the absorption coefficients $\alpha$. In Fig. 1 we plot absorption coefficients for $C=1$ and 3 for the two separate boundary conditions. Increasing the value of this constant $C$, narrows the kernel, resulting in a smaller value for the parameter $N$ (see Eqs. (26) and (27)). This clearly reduces the amount of p-mode absorption for the simulated plage. Here we have assumed that each flux tube in the plage is identical with the same $\beta(=1)$ value.

Clearly the absorption coefficient is sensitive to the width of the kernel function. Knowing accurate kernel functions used for observationally measured $\alpha$ in magnetic regions is thus crucial in correctly interpreting the amount of absorption observed.

\subsection{Effect of a random collection of flux tubes on absorption coefficients}

Jain et al. (2009) assumed that each flux tube in the simulated plage had the same value of $\beta$. However, intuitively, this is unlikely to be the case for real solar plage regions. Thus, here we consider magnetic fibrils with a random distribution of $\beta$ values, ranging from 0 to 2 as discussed in Sect. 4.3. We use three different random distributions, uniform, normal (Gaussian), and an exponential distribution and plot our results in Fig. 2. We generated 40 separate realisations for a given distribution and then plotted the mean absorption coefficient for this ensemble of realisations (solid curve). The error bars represent the standard deviation of $\alpha$ within these 40 realisations. For comparison we have also over-plotted, with dashed lines, the case of a simulated plage where each flux tube is now assumed to have same $\beta$, i.e., a collection of identical tubes with $\beta$ equalling the mean $\beta$ of the comparison distribution.

For maximal-flux BC, the solid and dashed curves are in good agreement with each other, whereas the stress-free BC shows larger deviations. This is due to the fact that the absorption 
A. Gascoyne et al.: Sensitivity of p-Mode Absorption
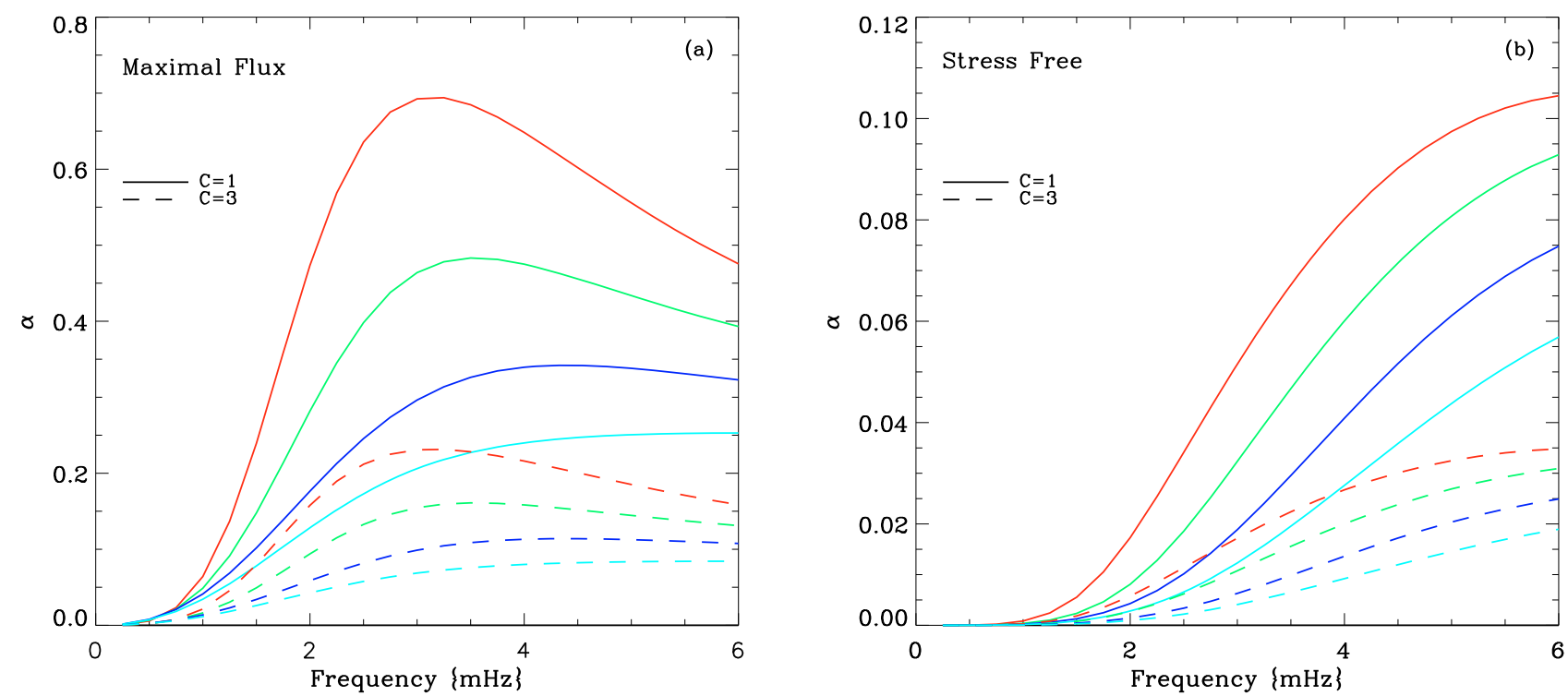

Fig. 1. Absorption coefficient calculated as a function of frequency for a simulated plage consisting of a number of flux tubes for two different BC. Each tube is assumed to be of the same $\beta(=1)$. Each panel shows the absorption coefficient for two different kernel function. One has much broader wings $(C=1)$ than the other $(C=3)$. Each mode order is represented by a different colour: red (p1), green (p2), blue (p3) etc.
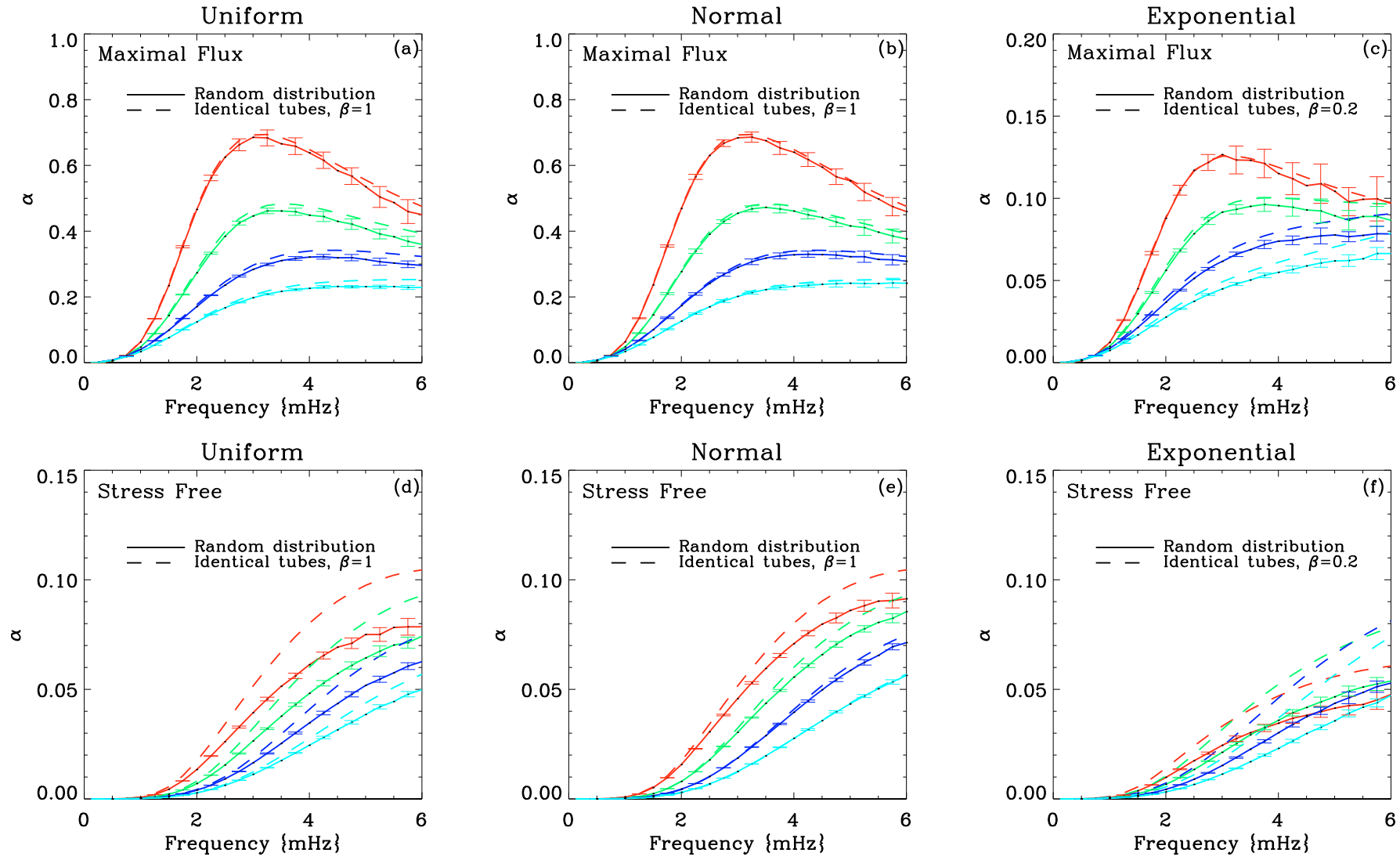

Fig. 2. Mean absorption coefficient with error bars calculated as a function of frequency for a simulated plage, for various random distributions in $\beta$. Over-plotted with dashed lines is the case for simulated plage with identical tubes of $\beta$ equal to the mean $\beta$ of the distribution. Each mode order is denoted by a different colour: red (p1), green (p2), blue (p3), etc.

coefficient is almost linear with $\beta$ (for $\beta<2$ ) for maximal-flux $\mathrm{BC}$, whereas for the stress-free $\mathrm{BC}$, it is nonlinear (this is explicitly explored in Jain et al. 2010). This effect is more for low radial orders since these waves are confined closer to the surface where the dynamics are more dominated by the upper boundary condition.
In Fig. 2 the error bars also show an interesting pattern, for low frequencies the error bars are very small and increase with frequency. The error bars also reduce with increasing mode order. This behaviour is due to the parameter $N$ (the number of tubes required), which, because of its dependence on the kernel, decreases as the horizontal wavenumber increases. The 
horizontal wavenumber increases with frequency and decreases with mode order. For high value for the number $N$ we have a larger sample size and thus lower errors, and vice versa for low $N$.

For the two upper BC used here, the maximal-flux condition produces the most promising results at a first glance when comparing with observations (see Braun \& Birch 2008). The stressfree condition lacks the fall off as seen in the observed absorption coefficients at higher frequencies. However the unphysical nature of this fall off (see Braun \& Birch 2008) suggests that extra caution should be taken when interpreting the observations. Also, in reality the actual reflection at the solar photosphere is likely to lie in between these two extreme conditions.

\section{Conclusions}

In this paper we have presented a calculation for the absorption coefficient for a collection of vertical, axisymmetric, thin magnetic flux tubes designed to simulate a solar plage region. The absorption mechanism used here is the generation of longitudinal (sausage) tube waves through buffeting of the magnetic fibrils by external solar $p$ modes. The generation of these tube waves carry energy out of the p-mode cavity and thus result in the absorption of $p$ modes.

We infer that there are a number of factors to consider when investigating the absorption of $\mathrm{p}$ modes by magnetic regions. In particular, the effect of the upper boundary condition, the observational kernel used to estimate the "effective" number of tubes in a plage and the distribution of plasma- $\beta$ amongst the collection of flux tubes. Besides local emission at high frequencies, other factors such as the amount of reflection from the surface, the plasma properties of the flux tubes in an ensemble and the form of the spacial weighting function all have considerable effect on the absorption coefficient.

The small size of the error bars (see Fig. 2) tells us that given the observed absorption coefficient for an active region, we can be confident on the likely distribution of $\beta$ that best fits this observed active region. Thus if one can obtain a good match between theory and observations we have a good diagnostic tool with which to measure the statistical properties of the flux tubes within a plage.

Acknowledgements. This work is supported by UK STFC studentship.

\section{References}

Abramowitz, M., \& Stegun, I. A. 1964, Handbook of Mathematical Functions (New York: Dover)

Bogdan, T. J., \& Cally, P. S. 1995, ApJ, 453, 919

Bogdan, T. J., Hindman, B. W., Cally, P. S., \& Charbonneau, P. 1996, ApJ, 465, 406

Braun, D. C., \& Birch, A. C. 2008, Solphys., 251, 267

Braun, D. C., Duvall, T. L., \& LaBonte, B. J. 1988, ApJ, 335, 1015

Cally, P. S., Crouch, A. D., \& Braun, D. C. 2003, MNRAS, 346, 381

Couvidat, S., Birch, A. C., \& Kosovichev, A. G. 2006, ApJ, 640, 516

Crouch, A. D., \& Cally, P. S. 2003, Sol. Phys., 214, 2011

D'Silva, S. 1994, ApJ, 435, 881

Gordovskyy, M., Jain, R., \& Hindman, B. W. 2009, ApJ, 694, 1602

Hanasoge, S. M., \& Cally, P. S. 2009, ApJ., 697, 651

Hindman, B. W., \& Jain, R. 2008, ApJ, 677, 769

Hollweg, J. V. 1988, ApJ, 335, 1005

Jain, R., Hindman, B. W., Braun, D. C., \& Birch, A. C. 2009, ApJ, 695, 325

Jain, R., Gascoyne, A., \& Hindman, B. W. 2010, MNRAS, submitted

Maltby, P., Avrett, E. H., Carlsson, M., et al. 1986, ApJ, 306, 284

Spruit, H. C. 1981, A\&A, 98, 155

Spruit, H. C. 1991, in Lecture Notes in Physics, ed. J. Toomre, \& D. O. Gough, Challenges to Theories of the Structure of Moderate Mass Stars (Berlin: Springer-Verlag), 388, 121 